\title{
Die Verbreitung des Doppelernährer- und Doppelbetreuermodells in fünf Ländern Europas
}

\author{
Ausgangspunkt unserer Analysen ist das Argument einer Reihe von Studien, dass die \\ Gleichheit der Geschlechter am besten verwirklicht ist, wenn beide Eltern sich in gleichem \\ Ausmaß am Arbeitsmarkt und an der Kinderbetreuung beteiligen. Der Beitrag beschreibt \\ Trends im sogenannten Doppelernährermodell (beide Eltern arbeiten Vollzeit) in \\ Deutschland, Frankreich, Großbritannien, Österreich und Spanien von 1998 bis 2010 und \\ untersucht die Aufteilung der Kinderbetreuungszeit in diesen Paarhaushalten.
}

CAROLINE BERGHAMMER, ROLAND VERWIEBE

\section{Einleitung}

In westlichen Gesellschaften gibt es eine wachsende Ausdifferenzierung von Modellen der Arbeitsteilung zwischen Eltern. Unter diesen ist die Gleichheit der Geschlechter im Doppelernährer- ${ }^{1} /$ Doppelbetreuermodell am weitesten fortgeschritten. Eltern überwinden hier die traditionelle geschlechtsspezifische Arbeitsteilung durch gleiche Teilhabe am Erwerbsleben und an der Kinderbetreuung. Dieses Modell hat den am stärksten geschlechtergerechten Charakter und wird von einigen Autoren als ein Ideal gepriesen. Gornick und Meyers sind hierfür ein Beispiel. Sie haben ein Bild einer modernen und fairen Gesellschaft entworfen, "[which] supports equal opportunities for men and women in employment, equal contributions from mothers and fathers at home, and high-quality care for children provided both by parents and well-qualified and well-compensated nonparental caregivers" (Gornick/Meyers 2008, S. 314f.). Ähnlich äußerten sich unlängst Esping-Andersen und Kollegen. Sie postulieren, dass sich moderne Gesellschaften mittelfristig in Richtung geschlechtergerechterer Strukturen entwickeln: „'Ideal typically', this entails partnerships based on two full-time employed spouses who engage in a gendersymmetric allocation of child care and housework" (EspingAndersen et al. 2013, S. 1282).

Warum nun ist das Doppelernährer-/Doppelbetreuermodell attraktiver als andere Modelle, in denen eine nach
Geschlecht spezialisierte Aufteilung der Arbeit vorherrscht? Neben der Gleichberechtigung als Ziel an sich zählen zu den Vorteilen eines solchen Modells die positiven Effekte (z. B. gesteigertes Wohlbefinden) eines erfolgreichen Rollenhandelns in der Arbeitssphäre und der häuslichen Sphäre (Carlson et al. 2006). Studien legen nahe, dass Fähigkeiten von der einen auf die andere Sphäre übertragen werden, oder dass auftretende Belastungen wechselseitig abgefedert werden können (Greenhaus/Powell 2006). Bestimmte Risiken, denen zum Beispiel geringfügig oder nicht beschäftigte Frauen ausgesetzt sind - Armut unter Alleinerziehenden und im Alter, das Stocken der Berufskarriere -, werden im Doppelernährer-/Doppelbetreuermodell abgeschwächt. Auch für Männer kann es Vorteile geben. Sie sind von der Bürde des Alleinernährers befreit und können sich stärker an der Kindererziehung beteiligen (Poortman/van der Lippe 2009; Gershuny 2013).

Ziel der Analyse ist die Untersuchung der Verbreitung des Doppelernährermodells und des Doppelbetreuermodells in fünf europäischen Staaten, die verschiedene Familienpolitiken repräsentieren: Deutschland, Frankreich, Großbritannien, Österreich und Spanien. Hierfür untersuchen wir die Entwicklung von Doppelernährer-Familien

1 Wir definieren Doppelernährer als duale Vollzeit-Verdiener mit jeweils 30 und mehr Wochenarbeitsstunden. 
über den Zeitraum von 1998 bis 2010. Paare werden als Doppelernährer eingeordnet, wenn beide Partner in Vollzeit arbeiten, d.h. 30 oder mehr Wochenarbeitsstunden (vgl. Fn. 1). Weiterhin analysieren wir die Aufteilung der Kinderbetreuungszeit in Doppelernährerpaaren. In Anlehnung an Pfau-Effingers Typologie des Doppelernährermodells unterscheiden wir zwischen dem Doppelbetreuermodell und dem Modell der außerhäuslichen Betreuung (Pfau-Effinger 2000). Während im ersten Modell die Aufgabe der Kinderbetreuung bei den Eltern liegt und partnerschaftlich zwischen Mutter und Vater aufgeteilt wird, wird die Kinderbetreuung im zweiten Modell stärker vom Staat, dem Markt oder dem Non-Profit-Sektor übernommen. Zusätzlich berücksichtigen wir ein weibliches und ein männliches Betreuermodell, in welchen entweder die Mutter oder der Vater den überwiegenden Anteil der Kinderbetreuungsarbeit leistet. Unsere Analyse basiert auf Daten der Arbeitskräfteerhebungen der Europäischen Union (EU) sowie auf Zeitbudgetdaten. Zur Berücksichtigung der Veränderungen im Lebenslauf haben wir alle empirischen Analysen abschnittsweise für den Familienlebenszyklus durchgeführt, operationalisiert am Alter des jüngsten Kindes.

Inwiefern können wir mit unserem Forschungsdesign die Befunde vorliegender Studien ergänzen? Die meisten Studien untersuchen die Verbreitung von Doppelernähreroder Doppelverdienermodellen ${ }^{2}$ in einzelnen Ländern, in Teilen wird auch ländervergleichend gearbeitet (Smith 2005; van Gils/Kraaykamp 2008; Kitterød/Rønsen 2012). Allerdings haben bisher wenige Studien gleichzeitig auch die Strukturen der Betreuung von Kindern systematisch mit in solche Analysen einbezogen. Unsere Studie ergänzt diese Literatur durch eine systematische länder-übergreifende Analyse von Doppelernährer-/Doppelbetreuer-Haushalten, in der wir auch eine Lebenslaufperspektive einbeziehen. Aus der vergleichenden Perspektive können wir einschätzen, ob der Aufstieg der Doppelernährer-Familien ein universeller, länderübergreifender Trend ist - wie in Teilen der Literatur angenommen (Esping-Andersen et al. 2013) -, oder ob eher (national-)spezifische Entwicklungen beobachtbar sind. Aus der Lebenslaufperspektive können wir konkretere Muster der Übernahme des Doppelernährermodells nach der Geburt eines Kindes untersuchen. Des Weiteren zeigt dieser Artikel, wie Doppelernährer-Eltern die Kinderbetreuung unter sich aufteilen und beleuchtet die sozialen Merkmale der Familien, in denen Doppelbetreuung der Kinder vorherrscht.

\section{Empirische Trends und theoretische Überlegungen}

Der Anstieg der Berufstätigkeit von Frauen in den letzten Jahrzehnten führte zur Zunahme von Doppelverdienerhaushalten und zur Annäherung ihrer Verbreitung in vielen europäischen Staaten. Dennoch beobachten wir länderübergreifend Abweichungen, vor allem befördert durch Unterschiede in der Arbeitsmarktintegration von Müttern. Diese Unterschiede sind am größten, wenn das jüngste Kind unter drei Jahren alt ist. Das entsprechende Spektrum ist weit gefächert, nur circa $10 \%$ der Mütter mit Kleinkindern sind in Ungarn und Tschechien am Arbeitsmarkt aktiv, während es mehr als $60 \%$ in den Niederlanden und in Belgien sind (Daten beziehen sich auf 2008; OECD 2013). Darüber hinaus gibt es deutliche Unterschiede bei den Arbeitszeiten: Teilzeitarbeit ist am weitesten verbreitet in den Niederlanden, in den deutschsprachigen Ländern und in Großbritannien, während in Zentral- und Osteuropa eher Vollzeitbeschäftigung vorherrscht (OECD 2013).

Die Praxis eines Doppelverdienermodells impliziert nicht notwendigerweise eine Gleichberechtigung zwischen den Partnern hinsichtlich beruflicher Arbeit auf der einen Seite und Haushalts- und Familienarbeit auf der anderen Seite. Sind beide Partner in den Arbeitsmarkt integriert, tendieren Frauen dazu, weniger Stunden als ihre männlichen Partner zu arbeiten (Hall/MacDermid 2009; Kitterød/ Rønsen 2012), und wenn beide Partner in Vollzeit arbeiten, verdienen Männer mehr als Frauen (Smith 2005). Ebenso impliziert doppeltes Einkommen nicht, dass der Karriere der Partner die gleiche Bedeutung beigemessen wird. Frauen machen eher Kompromisse als Männer, deren Karrieren (familienintern) immer noch häufig Vorrang eingeräumt wird (Solga/Wimbauer 2005).

Das Vordringen von Frauen auf den Arbeitsmarkt führte zu einer Neuverteilung der Hausarbeit unter Partnern, wenn auch (noch) nicht zu gleicher Aufteilung (Hook 2006). Die Zeit, die Eltern für die Kinderbetreuung aufbringen, nahm in den letzten Jahrzehnten zu, mit einer größeren relativen Zunahme bei den Vätern (Gershuny 2000; Gauthier et al. 2004). Eine ausgewogene Aufteilung von unbezahlter Arbeit zwischen den Partnern ist am wahrscheinlichsten in Doppelernährerhaushalten (Craig/Mullan 2011). Darüber hinaus ist in solchen Haushalten die insgesamt für die Kinderbetreuung aufgebrachte Zeit am geringsten, wobei ein größerer Anteil den Routinetätigkeiten zufällt, wie z. B. körperlicher Pflege. Die stärker ausgeglichene Aufteilung der Zeit für die Kinderbetreuung resultiert aus der Anpassung der Mütter an deren berufliche Anforderungen (d.h. Reduzierung der Kinderbetreuungszeit bei Vollzeitarbeit) und weniger aus der väterlichen Kompensation der Arbeitszeit der Mütter (Kitterød/Pettersen 2006). Bei Doppelernährer-Eltern ist die Arbeitslast von Lohnarbeit und Haus- und Familienarbeit insgesamt höher als in anderen Beschäftigungskonstellationen. Die Gesamtstunden-

2 Doppelverdienerpaare werden so definiert, dass beide Partner mindestens eine Stunde pro Woche am Arbeitsmarkt aktiv sind. Damit liegt das Arbeitsangebot der Haushalte in der Regel deutlich unter dem von Doppelernährerpaaren, dem Fokus unserer Studie. 
zahl der Mütter übersteigt die ihrer männlichen Partner und sie investieren einen höheren Anteil in Hausarbeit und Kinderbetreuung (Milkie et al. 2009; Berghammer 2013). Alle bislang vorliegenden Studien legen nahe, dass berufstätige Mütter nach der täglichen Berufstätigkeit eine „zweite Schicht" zuhause absolvieren (Hochschild 1989). Nicht nur widmen sie mehr Zeit der Haus- und Familienarbeit, auch passen sie ihre berufliche Planung eher den vordringlichen Anforderungen der Kindererziehung an, während Männer diese in ihrer (außerberuflichen) Freizeit absolvieren (Maume 2008).

Diese bisher vorgestellten Muster der (Re-)Organisation von Erwerbsarbeit und Familienarbeit waren zuletzt auch Gegenstand von theoretisch orientierten Beiträgen, in denen versucht wurde, die empirischen Trends des Aufkommens von Doppelernährer-Familien und deren gleichberechtigtere Aufteilung von Haus- und Familienarbeit zu erklären. Die ökonomische Theorie erklärt die Zunahme von weiblichen Beschäftigten mit deren höherem Einkommens-potenzial, welches - aufgrund der stark wachsenden Zahl hoher Bildungsabschlüsse von Frauen - dem der Männer zunehmend gleichkommt (Becker 1981). Die verbesserte Integration von Frauen in den Arbeitsmarkt geht einher mit sich verschlechternden Arbeitsmarktaussichten für (gering qualifizierte) Männer (Oppenheimer 1994; Giesecke/ Verwiebe 2010). Die Ressourcen, die Frauen aus Lohnarbeit beziehen, erhöhen aus theoretischer Sicht deren Verhandlungsmacht in der Beziehung. Die Verhandlungstheorie argumentiert in diesem Kontext, dass Partner unterschiedliche, wenn nicht sogar konkurrierende Präferenzen haben und untereinander aushandeln müssen. Erweiterte Ressourcen (z.B. Einkommen, Ausbildung) geben dem einen oder anderen Partner eine vorteilhafte Position (Bittman et al. 2003). Die Grundannahme ist, dass Menschen sich aus Hausarbeit „heraus zu verhandeln“ bestrebt sind, da diese generell als unbefriedigende Tätigkeit empfunden wird (Bianchi et al. 2000), wenngleich das bei der Kindererziehung weniger klar ist, da diese tendenziell positiv erlebt wird (Gershuny 2013). Daraus folgt die Annahme, dass Doppelernährer die Hausund Familienarbeit zu gleichmäßigeren Teilen organisieren als Paare, in denen die Frau weniger Stunden arbeitet. Dass die Aufteilung unbezahlter Arbeit dennoch eher zum Nachteil der Frauen tendiert, erklärt sich vor allem aus der weiterhin höheren Entlohnung von Männern und den traditionellen Erwartungen hinsichtlich der elterlichen Rollen.

\section{Strukturdaten der Untersuchungsländer}

Mit Deutschland (D), Frankreich (F), Großbritannien (GB), Österreich (AT) und Spanien (ES) betrachten wir Doppelernährerhaushalte in fünf Ländern, die sich hinsichtlich Familienpolitik, ökonomischen Bedingungen und Einstel- lungen zur Müttererwerbstätigkeit teilweise deutlich voneinander unterscheiden. Die Familienpolitik in Deutschland, Frankreich und Österreich basiert auf konservativen wohlfahrtsstaatlichen Prinzipien und den damit verbundenen, in vielen Bereichen relativ umfassenden Sozialleistungen. Großbritannien ist ein Beispiel für einen liberalen Wohlfahrtsstaat. Ähnlich wie in Spaniens mediterranem Wohlfahrtssystem ist das Niveau sozialer Fürsorge dort niedrig (Mau/Verwiebe 2010).

Die Daten in Tabelle 1 verweisen auf Unterschiede zwischen den Ländern in wichtigen Strukturbereichen. Die Dauer der bezahlten Elternzeit unterscheidet sich relativ stark, am längsten ist sie in Österreich und Frankreich, wo bis zum dritten Geburtstag des Kindes Elterngeld bezogen werden kann. In Großbritannien und Deutschland ist die Dauer mit maximal zehn bzw. 14 Monaten deutlich kürzer. Spanien bietet hingegen keine bezahlte Elternzeit. Mit Blick auf die Kinderbetreuungsquoten sind Frankreich, Großbritannien und Spanien positiv hervorzuheben. Im Ländervergleich ist hier vor allem die Situation von Familien mit Kindern unter drei Jahren vergleichsweise günstig. Diese ist für österreichische Familien mit Kleinkindern hingegen sehr problematisch, es gibt kaum Betreuungsmöglichkeiten. Auch mit Bezug auf die Einstellungen zur Berufstätigkeit von Müttern finden wir charakteristische Differenzen zwischen den Untersuchungsländern. So gibt beispielsweise in Österreich 62 \% der Bevölkerung an, dass ein Vorschulkind unter der Berufstätigkeit der Mutter leidet, in Westdeutschland sind dies $60 \%$ (Ostdeutschland $33 \%$ ), in Spanien $44 \%$, in Frankreich und Großbritannien nur $34 \%$ der Bevölkerung. Allerdings: In fast allen Ländern geht die Ablehnung gegenüber der Berufstätigkeit von Müttern zurück.

Mit Blick auf Arbeitsmarktcharakteristika lässt sich in allen Untersuchungsländern ein sehr starker Anstieg der Beschäftigung von Frauen beobachten (besonders in Spanien), während die Erwerbsquoten der Männer eher stagnieren. Damit korrespondierend beobachten wir einen Rückgang der Arbeitslosigkeit bei Frauen und einen Anstieg der Arbeitslosigkeit bei den Männern. Es ist anzumerken, dass dieses Beschäftigungsplus bei den Frauen maßgeblich durch eine Ausweitung der weiblichen Teilzeittätigkeiten getragen wird. Besonders deutliche Zunahmen beobachten wir im Untersuchungszeitraum in Deutschland und Österreich. In Großbritannien und Frankreich lässt sich keine vergleichbare Ausweitung der Teilzeitbeschäftigung feststellen.

Aus dem Dargelegten folgern wir, dass sich keines der Länder eindeutig in Richtung eines Doppelernährermodells bewegt, obgleich sich ändernde Einstellungen und Verbesserungen bei der institutionalisierten Kinderbetreuung ein solches Modell (zumindest mittelfristig) zu befördern scheinen. Die diskutierten Arbeitsmarktcharakteristika (Stichwort wachsende Teilzeittätigkeit) deuten vielmehr auf einen generellen Anstieg von Doppelverdiener-Familien hin. 


\section{Länderüberblick: Elternzeit, Kinderbetreuung, Einstellungen und Arbeitsmarktcharakteristika}

Angaben in absoluten Zahlen und in Prozent

\begin{tabular}{|c|c|c|c|c|c|c|c|c|c|c|c|c|c|c|c|c|c|c|c|c|}
\hline \multirow[t]{2}{*}{ Land } & \multicolumn{2}{|c|}{$\begin{array}{l}\text { Maximale Dauer } \\
\text { der bezahlten } \\
\text { Elternzeit in } \\
\text { Monaten (a) }\end{array}$} & \multicolumn{2}{|c|}{$\begin{array}{l}\text { Anteil der } \\
0-2-\text {-Jährigen in } \\
\text { institutionalisier- } \\
\text { ter Kinderbetreu- } \\
\text { ung (in Klammern: } \\
30+\text { Stunden pro } \\
\text { Woche) (b) }\end{array}$} & \multicolumn{2}{|c|}{$\begin{array}{l}\text { Anteil der } \\
3-5 \text {-Jährigen in in- } \\
\text { stitutionalisierter } \\
\text { Kinderbetreuung } \\
\text { (in Klammern: } 30+ \\
\text { Stunden pro } \\
\text { Woche) (c) }\end{array}$} & \multicolumn{2}{|c|}{$\begin{array}{l}\text { Vorschulkind lei- } \\
\text { det, wenn Mutter } \\
\text { arbeitet: stimme } \\
\text { zu + stimme sehr } \\
\text { zu (d) }\end{array}$} & \multicolumn{2}{|c|}{$\begin{array}{l}\text { Erwerbsquote, } \\
\text { 15-64 Jahre, } \\
\text { Männer (e) }\end{array}$} & \multicolumn{2}{|c|}{$\begin{array}{l}\text { Erwerbsquote, } \\
15-64 \text { Jahre, } \\
\text { Frauen (f) }\end{array}$} & \multicolumn{2}{|c|}{$\begin{array}{l}\text { Teilzeitquote, } \\
\text { Männer (g) }\end{array}$} & \multicolumn{2}{|c|}{$\begin{array}{l}\text { Teilzeitquote, } \\
\text { Frauen (h) }\end{array}$} & \multicolumn{2}{|c|}{$\begin{array}{c}\text { Arbeitslosenrate, } \\
\text { Männer (i) }\end{array}$} & \multicolumn{2}{|c|}{$\begin{array}{l}\text { Arbeitslosenrate } \\
\text { Frauen (j) }\end{array}$} \\
\hline & 1998 & 2010 & 2005 & 2010 & 2005 & 2010 & 1999 & 2008 & 1998 & 2010 & 1998 & 2010 & 1998 & 2010 & 1998 & 2010 & 1998 & 2010 & 1998 & 2010 \\
\hline D & 24 & $12(+2)$ & $16(8)$ & $20(13)$ & $86(26)$ & $92(46)$ & $53,7^{*}$ & $47,0^{* \prime}$ & 79,2 & 82,3 & 62,1 & 70,8 & 4,2 & 8,7 & 36,1 & 45,0 & 8,9 & 7,5 & 10,2 & 6,6 \\
\hline $\mathbf{F}$ & 36 & 36 & $32(16)$ & $42(26)$ & 95 (39) & $94(47)$ & 53,5 & 33,9 & 75,1 & 74,9 & 61,6 & 66,1 & 5,5 & 6,4 & 31,6 & 30,0 & 9,4 & 9,3 & 12,4 & 10,1 \\
\hline GB & 4.5 & 10 & $29(5)$ & $35(4)$ & $88(24)$ & $90(22)$ & 43,0 & 34,1 & 82,8 & 81,7 & 66,9 & 69,4 & 7,7 & 11,0 & 44,2 & 42,4 & 6,8 & 8,6 & 5,3 & 6,8 \\
\hline AT & $18(+6)$ & $30(+6)$ & $4(0)$ & $9(3)$ & 69 (16) & $83(26)$ & - & 61,8 & 80,2 & 80,9 & 62,5 & 69,3 & 4,0 & 7,8 & 30,0 & 43,3 & 3,8 & 4,6 & 5,4 & 4,2 \\
\hline ES & \multicolumn{2}{|c|}{$\begin{array}{c}\text { Keine bezahlte } \\
\text { Elternzeit }\end{array}$} & 39 (14) & $38(18)$ & $94(40)$ & $95(50)$ & 43,2 & 43,9 & 77,0 & 80,7 & 48,6 & 65,9 & 2,9 & 5,2 & 17,0 & 23,1 & 11,7 & 19,7 & 22,6 & 20,5 \\
\hline
\end{tabular}

Anmerkungen und Quellen:

(a) Inkludiert Mutterschutz nach der Geburt eines Kindes; Zahlen in Klammern geben die zusätzlichen Monate an, wenn sich die Eltern die Elternzeit teilen; Informationen zu 1998 aus Waldfogel (2001),

Frankreich: unbezahlte Elternzeit für erstes Kind; Informationen zu 2010 aus der Multilinks Datenbank (http://www.multilinks-project.eu/), Frankreich: an Familien mit einem Kind wird Elterngeld in den ersten sechs Monaten nach Ende des Mutterschutzes bezahlt, Großbritannien: 39 Wochen bezahlter Mutterschutz und 2 Wochen bezahlte Väterkarenz;

(b) und (c) Eurostat Datenbank (basierend auf EU-SILC, Daten seit 2005 verfügbar);

(d) Europäische Wertestudie 1999 und 2008; Berechnungen der Autoren (gewichtete Analysen), Altersgruppe 18-69, Messung auf einer 4-stufigen Skala, * 35,5 in Ost- und 71,4 in Westdeutschland,

**33,2 in Ost- und 59,7 in Westdeutschland, 1999 in Österreich nicht vergleichbar, Umfrage für Großbritannien aus 2009;

(e) und (f) Eurostat Datenbank;

(g) und (h) Eurostat Datenbank, Anteil an den Erwerbstätigen (15-64 Jahre);

(i) und (j) Eurostat Datenbank.

\section{Daten, Messgrößen und Methode}

Unsere Analyse der Doppelernährer-Familien basiert auf den EU-Arbeitskräfteerhebungen (EU-LFS, Version 11). Die EU-LFS werden auf Haushaltsebene durchgeführt, allerdings sind detaillierte Informationen zur Zusammensetzung von Haushalten erst seit dem Jahr 1998 verfügbar, weshalb wir die mit diesem Jahr beginnende Periode abdecken (Eurostat 2012). Aus Datenschutzgründen stammen die deutschen Daten nur aus den Jahren seit 2002. Unser Sample enthält Paare mit leiblichen und nicht-leiblichen Kindern unter 15 Jahren. Sie wurden einbezogen, wenn die Mutter zwischen 20 und 54 Jahren alt ist. Die Dynamiken elterlicher Berufstätigkeit über den Familienlebenszyklus wurden durch die Aufgliederung aller Analysen im empirischen Teil nach dem Alter des jüngsten Kindes im Haushalt gewonnen. Der OECD-Klassifikation folgend, unterscheiden wir drei Kategorien: null bis zwei, drei bis fünf sowie sechs bis 14 Jahre alte Kinder (Kleinkinder, Vorschulkinder und Schulkinder). Paare werden als Doppelernährer klassifiziert, wenn beide Partner mindestens 30 Wochenarbeitsstunden arbeiten, übereinstimmend mit der OECDDefinition von Vollzeitarbeit. In Doppelverdiener-Paaren sind beide Eltern mindestens eine Stunde pro Woche auf dem Arbeitsmarkt aktiv. Personen im Mutterschutz und in Karenz wurden den Nichterwerbstätigen zugeordnet.

Im zweiten Teil der Untersuchung fanden Zeitbudgetdaten Anwendung, mit denen die Verteilung der Kinderbetreuungszeit analysiert wurde. Wir verwendeten harmo- nisierte Daten der Multinational Time Use Study (MTUS, Version M5.53) (Fisher et al. 2012) für alle Staaten außer für Österreich, das in diesem Datensatz nicht repräsentiert ist. Hier stützen wir uns auf einen nationalen Datensatz. Wir untersuchten jene europäische Staaten, für die seit 1998 Daten erhoben wurden und für die Informationen auf der Haushaltsebene vorliegen: Deutschland, Frankreich, Großbritannien, Österreich und Spanien. Die Befragten führten mit Stift und Papier Tagebuch, indem sie mit eigenen Worten ihre Aktivitäten desselben Tages notierten (für mehr Informationen zu den Länderstichproben siehe Übersicht 1). Eine Ausnahme bildete Frankreich, wo die Aktivitäten des jeweiligen Tages durch Hausbesuche erfasst wurden.

\section{ÜBERSICHT 1}

\section{Länderstichproben}

Angaben in absoluten Zahlen

\begin{tabular}{l|c|c|c|c|c}
\hline Land & $\begin{array}{c}\text { Stichproben- } \\
\text { größe }\end{array}$ & Jahr* & $\begin{array}{c}\text { Umfrage- } \\
\text { periode }\end{array}$ & $\begin{array}{c}\text { Anzahl der } \\
\text { Tagebuchtage }\end{array}$ & $\begin{array}{c}\text { Zeitintervall in } \\
\text { Minuten }\end{array}$ \\
\hline D & 449 & 2001 & $04 / 01-03 / 02$ & 3 & 10 \\
\hline F & 503 & 1998 & $02 / 98-02 / 99$ & 1 & 10 \\
\hline GB & 423 & 2001 & $06 / 00-09 / 01$ & 2 & 10 \\
\hline AT & 134 & 2008 & $03 / 08-04 / 09$ & 1 & 15 \\
\hline ES & 1645 & 2003 & $10 / 02-10 / 03$ & 1 & 10 \\
\hline
\end{tabular}

Anmerkung: * Bezieht sich auf das Jahr, in dem die Mehrheit der Interviews durchgeführt wurde. Quelle: Centre forTime Use Research (2013). 
Unser MTUS-Sample enthält Eltern mit (nicht-)leiblichen Kindern unter 15 Jahren. Einbezogen wurden Paare mit Müttern im Alter zwischen 20 und 54 und Vätern von 20 bis 59 Jahren. Die zwei Schlüsselvariablen waren das elterliche Erwerbsmodell und die Kinderbetreuungszeit. Übereinstimmend mit der obigen Klassifikation waren DoppelernährerEltern jene, die in Vollzeit arbeiteten. Wir verwendeten die Messgröße der Kinderbetreuungszeit als Haupttätigkeit, da sich dies über die Zeit und länderübergreifend als verlässlicher erwies als eine gleichzeitige Berücksichtigung von Kinderbetreuung als Nebentätigkeit (Fedick et al. 2005). Kinderbetreuung umfasst unter anderem folgende Tätigkeiten: Vorlesen, Spielen und Beaufsichtigung, Hausaufgabenbetreuung und Lernen sowie Körperpflege und Betreuung von kranken und pflegebedürftigen Kindern (Fisher et al. 2012). Wir differenzierten zwischen vier Typen von Betreuungsmodellen:

(1) im Doppelbetreuermodell bringen Mütter und Väter mehr als $40 \%$ und unter $60 \%$ der täglichen Gesamtbetreuungszeit auf,

(2) im weiblichen Betreuermodell übernehmen Mütter $60-100 \%$ der Kinderbetreuungszeit und Väter 0-40\%,

(3) im männlichen Betreuermodell übernehmen Väter $60-100 \%$ der Kinderbetreuungszeit und Mütter 0-40\% und

(4) im Modell der außerhäuslichen Betreuung verbringen beide Eltern zusammen weniger als 30 Minuten am Tag mit Kinderbetreuung als Haupttätigkeit.

Schließlich haben wir mit logistischen Regressionen berechnet, wie wahrscheinlich das Auftreten eines Doppelbetreuermodells - im Vergleich mit anderen Betreuungsmodellen (d.h. weibliches, männliches, außerhäusliches Betreuermodell) - in einem Doppelernährerhaushalt ist. Dafür haben wir eine gepoolte Stichprobe aller Länder genutzt. Die erklärenden Variablen in diesem Modell wurden wie folgt definiert: Das Alter der Mutter wurde in Jahren gemessen. Beim Altersunterschied zwischen Partnern gab es drei Kategorien:

- ungefähr gleichaltrig (der Mann ist zwei Jahre jünger bis drei Jahre älter als die Frau),

- Mann jünger (drei und mehr Jahre jünger) und

- Mann älter (vier oder mehr Jahre älter).

Die Ausbildung wurde dichotomisiert in:

- bis postsekundäre Bildung (ISCED 0 - 4) und

- tertiäre Bildung, abgeschlossen und gegenwärtiger Besuch

(ISCED 5-6).

Auf dieser Basis wurde die Ausbildung eines Paares kombiniert zu:

- beide geringer als tertiäre Bildung

- Mann tertiär, Frau geringer
- Frau tertiär, Mann geringer

- beide tertiäre Bildung.

Der Beruf der Frau wurde klassifiziert in vier Kategorien:

- (niedrig-)qualifizierte manuelle Tätigkeiten

- niedrig-qualifizierte Dienstleistungsberufe

- qualifizierte Dienstleistungsberufe

- Fachkräfte. ${ }^{3}$

Die Zahl der Kinder wurde dichotomisiert in ein Kind versus zwei und mehr Kinder, das Alter des jüngsten Kindes in null bis fünf Jahre und sechs bis 14 Jahre. Wochentage wurden unterschieden von Wochenenden. Des Weiteren wurde die Variable Land im Modell berücksichtigt, um Differenzen zwischen den Untersuchungsländern thematisieren zu können.

\section{Trends im Doppelernährermodell}

In diesem Abschnitt präsentieren wir für unsere Untersuchungsländer deskriptive Trends in Doppelverdienerund Doppelernährer-Familien unter Berücksichtigung des Alters des jüngsten Kindes (Abbildung 1). Beobachtbare Unterschiede zwischen diesen beiden Indikatoren erklären sich großteils aus der Verbreitung eines modernisierten männlichen Ernährermodells, in dem die Frau teilzeitbeschäftigt ist. In Deutschland gab es z. B. einen Aufwärtstrend bei Doppelverdiener-Familien, während die Ausprägung des Doppelernährer-Profils schwach blieb (mit Ausnahme von Eltern mit Vorschulkindern). Dies reflektiert - wie in Österreich - die gestiegene Unterstützung von Teilzeitarbeit. In Österreich beobachten wir einen starken Rückgang bei beiden Typen von Beschäftigungsmodellen unter Eltern mit Kleinkindern, was hauptsächlich durch die Verlängerung der Elternzeit im Jahr 2002 ausgelöst wurde (Berghammer 2014): Der Anteil von Doppelernährer-Familien verringerte sich um mehr als die Hälfte. Als eine Konsequenz verschärften sich die Unterschiede im Erwerbsverhalten zwischen Eltern mit Kindern unter und über drei Jahren. Bei Haushalten mit Vorschul- oder Schulkindern kam es zu einer stärkeren Verbreitung von Doppelverdiener-Familien

3 Die 14 Kategorien der harmonisierten Variable in der MTUS wurden wie folgt gruppiert (vgl. Fisher et al. 2012): Fachkräfte (1-7), qualifizierte Dienstleistungsberufe $(8-10,14)$, niedrig-qualifizierte Dienstleistungsberufe (11) sowie niedrig-qualifizierte und qualifizierte manuelle Arbeit. In Österreich wurde der Kategorisierung die Internationale Standardklassifikation der Berufe zugrunde gelegt: Fachkräfte $(1-2)$, qualifizierte Dienstleistungsberufe (0 und 3), niedrigqualifizierte Dienstleistungsberufe (4-5) sowie niedrig-qualifizierte und qualifizierte manuelle Arbeit (6-9). 
ABB. 1

\section{Anteil der Doppelverdiener und Doppelernährer nach Alter des jüngsten Kindes im Ländervergleich}

Angaben in Prozent

$$
\text { - Doppelernährer 0-2 Jahre } \quad \text { - Doppelverdiener 0-2 Jahre " Doppelverdiener 3-5 Jahre } \quad \text { " " " Doppelverdiener 6-14 Jahre }
$$
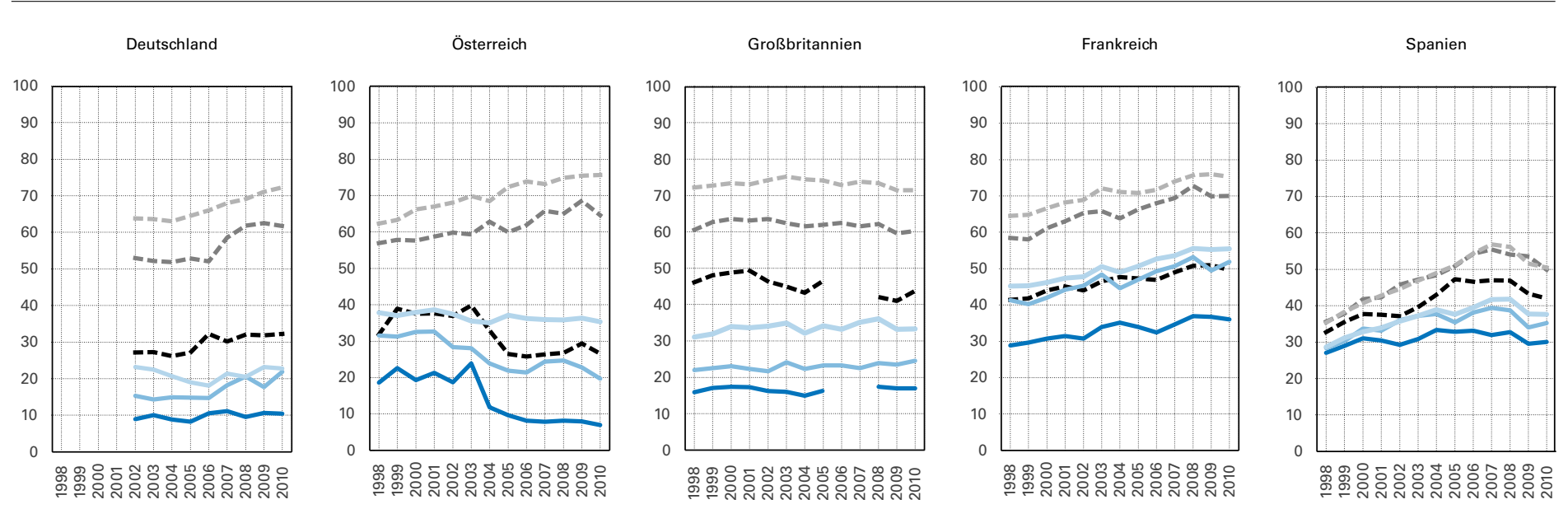

Anmerkungen: Doppelverdiener: beide Eltern sind beschäftigt; Doppelernährer: beide Eltern sind vollzeitbeschäftigt (30 und mehr Stunden). Aufgrund von Problemen mit der Datenqualität keine Berechnungen für Großbritannien in den Jahren 2006 und 2007 möglich (0-2 Jahre).

Quelle: EU-Arbeitskräfteerhebungen (gewichtete Analysen); Berechnungen der Autoren.

aufgrund der schnelleren Rückkehr der Mütter auf den Arbeitsmarkt. Analog ist Großbritannien gekennzeichnet durch ein vergleichsweise niedriges Niveau von Doppelernährer-Eltern und die Verfügbarkeit von Teilzeitarbeit. Abweichend von Österreich und Deutschland war die Erwerbsquote von Müttern in Großbritannien zu Beginn des Untersuchungszeitraums bereits hoch und blieb seither stabil, ebenso der Prozentsatz von DoppelernährerFamilien. Frankreich ist eine Ausnahme in der Untersuchungsgruppe. Es hat den höchsten Anteil von Doppelverdiener- und Doppelernährer-Familien. Französische Mütter kehren deutlich früher in ihre (Vollzeit-) Positionen zurück als Mütter in den anderen Ländern: Im Jahr 2010 war die Hälfte der Elternpaare mit Kleinkindern Doppelverdiener. Dieser Prozentsatz erhöhte sich noch für jene mit Vorschulkindern, blieb aber recht stabil für Paare mit Schulkindern. Spanien ähnelt Frankreich mit seinem hohen Anteil an Doppelernährern unter Eltern mit Kindern jeden Alters, aber es unterscheidet sich durch das geringe Gefälle zu den Doppelverdienern. Tatsächlich ist die Wahrscheinlichkeit, in Spanien auf Doppelverdiener-Eltern mit drei- bis 14-jährigen Kindern zu treffen, die geringste in allen fünf Ländern. Etwa ein Drittel der spanischen Mütter mit einem Kleinkind setzte ihre Vollzeittätigkeit fort. Allerdings verbessert sich die Arbeitsmarktintegration der spanischen Mütter nicht mehr substanziell, wenn die Kinder älter werden. Der Aufwärtstrend unter Doppelverdiener-Eltern kehrte sich im Zuge der Wirtschaftskrise um, die das Land am Ende des Betrachtungszeitraums hart getroffen hat.

\section{Verbreitung des Doppelernährer-/ Doppelbetreuermodells}

Abbildung 2 zeigt, in welchem Ausmaß das Doppelernährer-/ Doppelbetreuermodell vorkommt. Das Doppelbetreuermodell scheint insgesamt ein wenig verbreitetes Arrangement zu sein, es wird in unseren Untersuchungsländern lediglich von $20 \%$ der Doppelernährer-Eltern mit einem Kleinkind und von 15 \% der Familien mit einem Vorschulkind umgesetzt. Unter Eltern mit Kindern dieser Altersgruppen ist das weibliche Betreuermodell in allen fünf Ländern am häufigsten anzutreffen: Es betrifft $63 \%$ der Doppelernährer-Eltern mit einem Kleinkind und $57 \%$ jener mit einem Vorschulkind. Wenn das jüngste Kind die Schule besucht, praktizieren 52 \% der Doppelernährer-Eltern das Modell der außerhäuslichen Betreuung (in welchem die Betreuungszeit beider Eltern weniger als 30 Minuten pro Tag ausmacht). Die Wahrscheinlichkeit, dass beide Eltern die Betreuung zu gleichen Anteilen unter sich aufteilen, sinkt mit zunehmendem Alter des Kindes. Doch obwohl das Doppelbetreuermodell nur bei einer Minderheit der Doppelernährer-Paare Anwendung findet, ist es hier doch deutlich wahrscheinlicher als im traditionellen männlichen Ernährermodell (Mann arbeitet Vollzeit, Frau ist nicht erwerbstätig) und - mit einigen Ausnahmen - im modernisierten männlichen Ernährermodell, in dem Frauen in Teilzeit arbeiten. ${ }^{4}$

4 Die hier zugrunde liegende Tabelle ist aus Platzgründen nicht dargestellt, auf Anfrage bei den Autoren erhältlich. 
ABB. 2

\section{Betreuermodelle der Doppelernährerhaushalte nach Alter des jüngsten Kindes im Ländervergleich}

Angaben in Prozent

- Doppelbetreuer

- überwiegend Vater

überwiegend Mutter

- außerhäusliche Betreuung

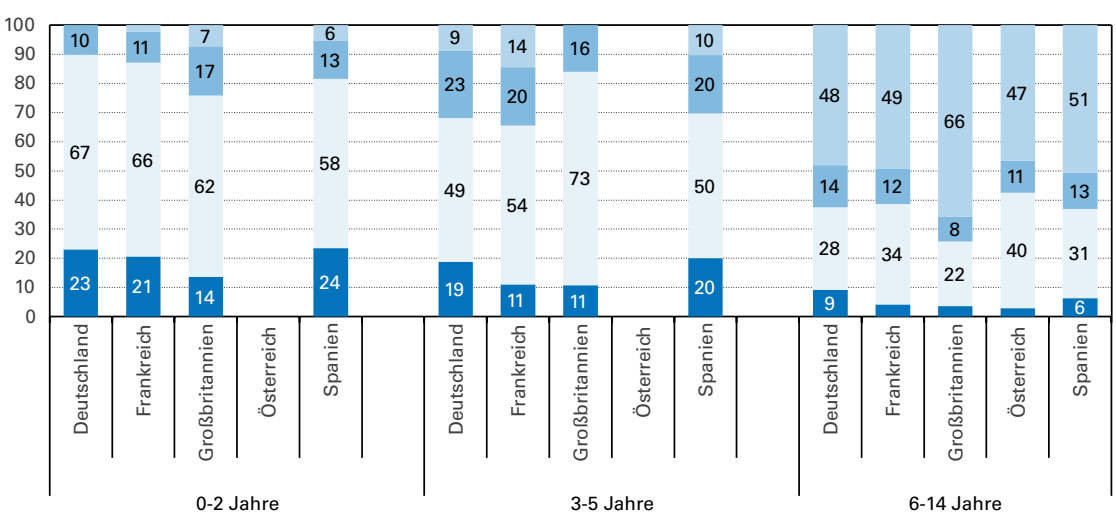

Anmerkung: Aufgrund zu geringer Fallzahlen keine Berechnungen für Österreich möglich (0-2 Jahre und 3-5 Jahre).

Quelle: Multinational Time Use Study (FR 1998, DE 2001, ES 2003, UK 2001) und Österreichische Zeitverwendungserhebung (2008); Berechnungen der Autoren. pelbetreuermodell häufiger zu beobachten, wenn beide Partner eine tertiäre Bildung besitzen. In Fällen, in denen der berufliche Status der Frauen hoch ist (hoch qualifizierte Fachkräfte und qualifizierte Dienstleistungsberufe), tendieren Paare ebenfalls zu einer gleichberechtigteren Aufteilung der Kinderbetreuung. Der familiäre Kontext zählt insofern, als Betreuungszeiten gleichmäßiger in Familien mit zwei oder mehr Kindern verteilt werden und in denen das jüngste Kind unter sechs Jahren ist; dies konnten wir bereits in den deskriptiven Analysen andeuten. Eltern teilen die Verantwortung für die Kinderbetreuung an Wochenenden fairer auf als über den Verlauf der Arbeitswoche. Nachdem in den Regressionsanalysen für demografische und sozialstrukturelle Merkmale auf der Paarebene kontrolliert wurde, finden wir signifikante Unterschiede zwischen Deutschland und Frankreich bzw. Großbritannien.

\section{Diskussion}

Als Ausgangspunkt unserer Analysen haben wir folgende Argumente einer Reihe von Studien gewählt:

- Moderne Gesellschaften sollten ein geschlechtergerechtes Doppelernährer-/Doppelbetreuermodell anstreben.

- Dieses Modell wird sich mittelfristig in Europa und Nordamerika durchsetzen (Gornick/Meyers 2008; Esping-Andersen et al. 2013).

Empirisch haben wir dies für insgesamt fünf europäische Staaten für den Zeitraum zwischen 1998 und 2010 untersucht. Dabei konnten wir eindeutig keinen einheitlichen Trend zum Doppelernährermodell feststellen. Während dieses Modell in Frankreich und Spanien weitere Verbreitung fand, stagniert es in Deutschland und Großbritannien, in Österreich gab es sogar einen Rückgang. Dagegen erfährt das Doppelverdienermodell wachsende Akzeptanz, wie die stärkere Verbreitung in den untersuchten Staaten zeigt.

Im Detail zeigt sich, dass das Leben in einem Doppelernährermodell nicht zu gleicher Aufteilung der Kinderbetreuung unter Eltern führt: Länderübergreifend übernehmen nur 18 \% der Familien mit zwei in Vollzeit arbeitenden Erwachsenen und einem Kind unter sechs Jahren das Doppelernährermodell, und lediglich $5 \%$ mit einem jüngsten Kind im Schulalter. Die Unterschiede zwischen Deutschland, Frankreich, Großbritannien, Österreich und Spanien sind diesbezüglich unerwartet gering, was unter Einbeziehung weiterer Untersuchungsländer (z. B. der nordischen Länder) weiter geprüft werden könnte (Esping-Andersen et al. 2013). In jedem Fall gibt es einen starken Kontrast zwischen der

5 Auch diese Tabelle ist aus Platzgründen nicht dargestellt und auf Anfrage bei den Autoren erhältlich. 
theoretischen Bedeutung des Doppelernährer-/Doppelbetreuermodells und seiner empirischen Häufigkeit. Stattdessen bleibt das weibliche Betreuermodell die häufigste Lebensweise unter Doppelernährer-Familien für Paare mit Vorschulkindern. Dies steht in Übereinstimmung mit Studien - einige von ihnen datieren zurück bis in die 1980er Jahre -, die gezeigt haben, dass Mütter nach der Arbeit am Abend eine „zweite Schicht” (Hochschild 1989) der Hausund Familienarbeit absolvieren. Väter neigen dazu, Aufgaben der Kinderbetreuung zu übernehmen, wenn diese ihre Berufstätigkeit nicht beeinträchtigen, vor allem am Wochenende. Der Fakt, dass Eltern gleichermaßen in den $\mathrm{Ar}-$ beitsmarkt involviert sind, impliziert also nicht, dass die Familienarbeit auf die Väter oder externe Betreuer übertragen wird. Folgerichtig ist die Arbeitslast von Müttern sehr hoch und übersteigt die der Väter in der Regel sehr deutlich (Milkie et al. 2009).

Eine weitere wichtige Erkenntnis unserer Studie bezieht sich auf die Bildung und den beruflichen Status von Eltern. In den untersuchten europäischen Ländern ist die Betreuungszeit gleichmäßiger verteilt, wenn beide Eltern eine tertiäre Bildung besitzen (siehe auch Craig/Mullan 2011) und wenn Mütter in Jobs mit hohem Status arbeiten. Eine faire Aufteilung von Aufgaben der Kinderbetreuung findet sich auch häufiger bei den Paaren, in denen die Frauen älter sind als die Männer. Diese Ergebnisse decken sich mit dem Argument der Verhandlungstheorie, dass größere (ökonomische) Ressourcen aufseiten der Frau zu einer egalitäreren Verteilung der unbezahlten Arbeit in der Familie führen (Bittman et al. 2003). Darüber hinaus ist es bei Paaren mit hoher Bildung und bei Paaren mit nicht-traditionellen Alterskombinationen wahrscheinlicher, dass moderne Einstellungen zur Rolle der Geschlechter vorherrschen.

Die noch immer weitgehend traditionelle Aufteilung von Betreuungsarbeit unter vollzeitbeschäftigten Erwachsenen kann aus unserer Sicht zumindest teilweise erklären, warum das Doppelernährermodell in Europa (noch) nicht weiter verbreitet ist, trotz wachsenden Bildungskapitals und höherer Verdienstmöglichkeiten bei Frauen. Angesichts der ungleichen Verteilung der Familienarbeit und der damit verbundenen Belastungen stellt das bestehende Arrangement eine schwere Bürde für das Zeitbudget und die Lebensqualität vieler Frauen dar.
TABELLE 2

\section{Einflussfaktoren auf das Auftreten eines Doppelbetreuermodells in Doppelernährerhaushalten}

Logistische Regression

\begin{tabular}{|c|c|c|}
\hline & Odds Ratio & $\begin{array}{l}\text { Verteilung der unabhän- } \\
\text { gigen Variablen (in \%) }\end{array}$ \\
\hline Alter der Mutter & $0,94 * * *$ & 37,7 (Mittelwert) \\
\hline \multicolumn{3}{|l|}{ Altersdifferenz zwischen den Partnern } \\
\hline Alter gleich (Ref.) & 1 & 63,1 \\
\hline Frau jünger & 0,97 & 30,1 \\
\hline Frau älter & $1,57^{*}$ & 6,9 \\
\hline \multicolumn{3}{|l|}{ Bildung der Eltern } \\
\hline Beide geringer als tertiäre Bildung (Ref.) & 1 & 47,0 \\
\hline Mann tertiär, Frau geringer & 0,99 & 11,5 \\
\hline Frau tertiär, Mann geringer & 1,09 & 16,2 \\
\hline Beide tertiäre Bildung & $1,32 \#$ & 25,4 \\
\hline \multicolumn{3}{|l|}{ Beruf der Frau } \\
\hline $\begin{array}{l}\text { (Niedrig-)qualifizierte manuelle Tätigkeiten } \\
\text { (Ref.) }\end{array}$ & 1 & 10,0 \\
\hline Niedrig-qualifizierte Dienstleistungsberufe & 1,14 & 20,8 \\
\hline Qualifizierte Dienstleistungsberufe & $1,66 \#$ & 34,4 \\
\hline Fachkräfte & $1,93 *$ & 34,8 \\
\hline \multicolumn{3}{|l|}{ Anzahl der Kinder } \\
\hline 1 (Ref.) & 1 & 45,4 \\
\hline $2+$ & $1,25 \#$ & 54,6 \\
\hline \multicolumn{3}{|l|}{ Alter des jüngsten Kindes } \\
\hline 0-5 Jahre (Ref.) & 1 & 40,3 \\
\hline 6-14 Jahre & $0,38 * * *$ & 59,7 \\
\hline \multicolumn{3}{|l|}{ Tag in der Woche } \\
\hline Wochentag (Ref.) & 1 & 65,4 \\
\hline Wochenende & $1,31^{*}$ & 34,6 \\
\hline \multicolumn{3}{|l|}{ Land } \\
\hline Deutschland (Ref.) & 1 & 14,2 \\
\hline Frankreich & $0,57^{*}$ & 16,0 \\
\hline Großbritannien & $0,38 * * *$ & 13,4 \\
\hline Österreich & 0,59 & 4,3 \\
\hline Spanien & 0,94 & 52,2 \\
\hline$n$ & 3154 & \\
\hline Pseudo (Nagelkerke) R2 & 0,118 & \\
\hline
\end{tabular}

Signifikanzniveau: ${ }^{* *} \mathrm{p}<0,001 ;{ }^{*} \mathrm{p}<0,01 ;{ }^{*} \mathrm{p}<0,05 ; \# \mathrm{p}<0,10$.

Quelle: Multinational Time Use Study (FR 1998, GE 2001, ES 2003, UK 2001) und Österreichische Zeitverwendungserhebung (2008); Berechnungen der Autoren. 


\section{LITERATUR}

Becker, G. S. (1981): A treatise on the family, Harvard

Berghammer, C. (2013): Keine Zeit für Kinder? Veränderungen in der Kinderbetreuungszeit von Eltern in Deutschland und Österreich, in: Zeitschrift für Sozio logie 42 (1), S. 52-73

Berghammer, C. (2014): The return of the male breadwinner model? Educational effects on parents' work arrangements in Austria, 1980-2009, in: Work

Employment and Society 28 (4), S. 611-632

Bianchi, S. M./Milkie, M. A./Sayer, L. C./Robinson, J. P. (2000): Is anyone doing the housework? Trends in the gender division of household labor, in: Social Forces 79 (1), S. $191-228$

Bittman, M./England, P./Sayer, L./Folbre, N./Matheson, G. (2003): When does gender trump money? Bargaining and time in household work, in: American Journal of Sociology 109 (1), S. 186-214

Carlson, D. S./Kacmar, K. M./Wayne, J. H./Grzywacz, J. G. (2006): Measuring the positive side of the work-family interface: Development and validation of a work-family enrichment scale, in: Journal of Vocational Behavior 68 (1),

S. $131-164$

Centre for Time Use Research (2013): http://www.timeuse.org/ (Januar 2013)

Craig, L./Mullan, K. (2011): How mothers and fathers share childcare: A crossnational time-use comparison, in: American Sociological Review 76 (6),

S. $834-861$

Esping-Andersen, G./Boertien, D./Bonke, J./Gracia, P. (2013): Couple specialization in multiple equilibria, in: European Sociological Review 29 (6),

S. $1280-1294$

Eurostat. (2012): EU Labour Force Survey database user guide

Fedick, C. B./Pacholok, S./Gauthier, A. H. (2005): Methodological issues in the estimation of parental time - Analysis of measures in a Canadian time-use survey, in: Electronic International Journal of Time Use Research 2 (1),

S. $67-87$

Fisher, K./Gershuny, J./Altintas, E./Gauthier, A. H. (2012): MultinationalTime Use Study user's guide and documentation, Oxford

Gauthier, A. H./Smeeding, T. M./Furstenberg, F. F., Jr. (2004): Are parents inves ting less time in children? Trends in selected industrialized countries, in: Population and Development Review 30 (4), S. 647-671

Gershuny, J. (2000): Changing times. Work and leisure in postindustrial society, Oxford

Gershuny, J. (2013): National utility: Measuring the enjoyment of activities, in: European Sociological Review 29 (5), S. 996-1009

Giesecke, J./Verwiebe, R. (2010): Erwerbschancen und Arbeitsmarktintegration im wiedervereinigten Deutschland, in: Krause, P./Ostner, I. (Hrsg.): Leben in Ost- und Westdeutschland. Eine sozialwissenschaftliche Bilanz der deutschen Einheit 1990-2010, Frankfurt a. M., S. 247-276

Gornick, J. C./Meyers, M. K. (2008): Creating gender egalitarian societies: An agenda for reform, in: Politics \& Society 36 (3), S. 313-349

Greenhaus, J. H./Powell, G. N. (2006): When work and family are allies: A theory of work-family enrichment, in: The Academy of Management Review 31

(1), S. $72-92$

Hall, S. S./MacDermid, S. M. (2009): A typology of dual earner marriages based on work and family arrangements, in: Journal of Family and Economic Issues 30 (3), S. 215-225

Hochschild, A. R. (1989): The second shift. Working families and the revolution at home, London

Hook, J. L. (2006): Care in context: Men's unpaid work in 20 countries, 1965-2003, in: American Sociological Review 71 (4), S. 639-660

Kitterød, R. H./Pettersen, S. V. (2006): Making up for mothers' employed working hours? in: Work, Employment \& Society 20 (3), S. 473-492

Kitterød, R. H./Rønsen, M. (2012): Non-traditional dual earners in Norway:

When does she work at least as much as he? in: Work, Employment \& Society 26 (4), S. 657-675

Mau, S./Verwiebe, R. (2010): European societies. Mapping structure and change, Bristol

Maume, D. J. (2008): Gender differences in providing urgent childcare among dual-earner parents, in: Social Forces 87 (1), S. 273-297

Milkie, M. A./Raley, S. B./Bianchi, S. M. (2009): Taking on the second shift: Time allocations and time pressure of U.S. parents with preschoolers, in: Social Forces 88 (2), S. 487-518

Oppenheimer, V. K. (1994): Women's rising employment and the future of the family in industrial societies, in: Population and Development Review 20 (2), S. $293-342$
Organisation for Economic Co-operation and Development (OECD) (2013): Family database, http://www.oecd.org/els/soc/oecdfamilydatabase.htm (Januar 2013)

Pfau-Effinger, B. (2000): Kultur und Frauenerwerbstätigkeit in Europa. Theorie und Empirie des internationalen Vergleichs, Opladen

Poortman, A.-R./van der Lippe, T. (2009): Attitudes toward housework and child care and the gendered division of labor, in: Journal of Marriage and Family 71 (3), S. 526-541

Smith, M. (2005): Dual earning in Europe:Time and occupational equity, in: Work, Employment \& Society 19 (1), S. 131-139

Solga, H./Wimbauer, C. (Hrsg.) (2005): „Wenn zwei das Gleiche tun ...“: Ideal und Realität sozialer (Un-)Gleichheit in Dual Career Couples, Opladen van Gils, W./Kraaykamp, G. (2008): The emergence of dual-earner couples: A longitudinal study of the Netherlands, in: International Sociology 23 (3), S. 345-366

Waldfogel, J. (2001): International policies toward parental leave and child care, in: The Future of Children 11 (1), S. 98-111

\section{AUTOREN}

CAROLINE BERGHAMMER, Dr., ist Universitätsassistentin am Institut für Soziologie der Universität Wien und Wissenschaftliche Mitarbeiterin am Wittgenstein Centre (IIASA, VID/ÖAW, WU), Vienna Institute of Demography/Österreichische Akademie der Wissenschaften. Arbeitsschwerpunkte: Religion und Fertilität, Erwerbsmodelle von Eltern, Zeitverwendung.

caroline.berghammer@univie.ac.at

ROLAND VERWIEBE, Dr., ist Professor für Soziologie und Vorstand des Instituts für Soziologie der Universität Wien. Arbeitsschwerpunkte: Arbeitsmarkt, soziale Ungleichheit, Armut, Migration, Europäisierung.

roland.verwiebe@univie.ac.at 